(C) The Authors

\title{
MEASUREMENT OF EXCITED HYPERONS IN PHOTOPRODUCTION AT CLAS*
}

\author{
KEI MORIYA $^{\dagger, \S}$ and REINHARD A. SCHUMACHER ${ }^{\ddagger}, \boldsymbol{\uparrow}$ \\ (for the CLAS Collaboration) \\ ${ }^{\dagger}$ Department of Physics, Indiana University, \\ Bloomington, IN 47405-7105, USA \\ ${ }^{\ddagger}$ Department of Physics, Carnegie Mellon University, \\ Pittsburgh, PA 15213, USA \\ \$kmoriya@indiana.edu \\ Ischumacher@cmu.edu
}

Published 14 January 2014

\begin{abstract}
Measurement results of photoproduced excited hyperon states using the CLAS detector at Jefferson Lab are shown. The invariant mass distribution of the $\Lambda(1405)$ has recently been shown to be different for each of the three $\Sigma \pi$ channels that it decays to, showing that there is prominent interference between the isospin $I=0$ and $I=1$ isospin amplitudes. Measurements of the differential and total cross sections of the three hyperons $\Lambda(1405), \Sigma^{0}(1385)$, and $\Lambda(1520)$ are presented and compared. Prospects of future studies using a $12 \mathrm{GeV}$ beam with the GlueX detector are briefly given.
\end{abstract}

Keywords: Hyperons; $\Lambda(1405) ; \Sigma(1385) ; \Lambda(1520)$; CLAS; GlueX; photoproduction.

\section{Introduction}

Photoproduction of excited hyperon states has not yet been studied in detail compared to the production of the ground state hyperons. It has been suggested that some of the so-called "missing" $N^{*}$ resonances may have relatively strong couplings to states besides $\pi N$, for example the $K^{+} Y$ states, which may lead to a solution to the missing baryon problem. Recent works ${ }^{1}$ show that indeed some of the resonances have couplings to the ground state $K^{+} \Lambda$ and $K^{+} \Sigma^{0}$ states.

While the coupling to intermediate $N^{*}$ states is an interesting problem, the nature of the excited hyperon states themselves is also an interesting topic, as are the photoproduction mechanisms. Below we present some of the recent excited hyperon measurements on the $\Lambda(1405), \Sigma^{0}(1385)$, and $\Lambda(1520)$.

*This is an Open Access article published by World Scientific Publishing Company. It is distributed under the terms of the Creative Commons Attribution 3.0 (CC-BY) License. Further distribution of this work is permitted, provided the original work is properly cited. 


\section{2. $\Lambda(1405)$ Line Shape Measurements}

The $\Lambda(1405)$ is a state of interest due to its peculiar $\Sigma \pi$ invariant mass spectrum, or "line shape". It has been suggested in chiral unitary coupled channel studies that the $\Lambda(1405)$ is a dynamically generated resonance, ${ }^{2}$ and a calculation within this framework predicted that each of the different $\Sigma \pi$ line shapes would be different. ${ }^{3}$ In recent years, there have been several measurements of the $\Lambda(1405)^{4}$ in various reactions, and this has lead to an exciting interplay between theory and experiment. Together with the $\Sigma(1385)$ and $\Lambda(1520)$, these are the lightest hyperon resonances, with spin-parity numbers of $1 / 2^{-}, 3 / 2^{+}$, and $3 / 2^{-}$, respectively. As each hyperon has different quantum numbers, a comparison of the production of the three states may reveal characteristics that are specific to the state or are otherwise common to the photoproduction mechanisms of each state.

The CLAS detector was located in Hall B of the Thomas Jefferson National Accelerator Facility (JLab), and utilized a real photon beam for photoproduction experiments. We report on the reactions $\gamma+p \rightarrow K^{+} Y^{*}$, where $Y^{*}$ is one of $\Lambda(1405)$, $\Sigma^{0}(1385)$, or $\Lambda(1520)$. The subsequent decays are into the $\Sigma \pi$ channels for the $\Lambda(1405)$ and $\Lambda(1520)$, and $\Lambda \pi^{0}$ for the $\Sigma^{0}(1385)$. Details of the analysis can be found in Refs. 5 and 6 . The line shapes of the $\Lambda(1405)$ in two center-of-mass (c.m.) energy bins $W$ are shown in Fig. 1.

Fits to extract the pole positions were performed in Ref. 7, and examples of the fit results are shown as the curves Fig. 1. The fits were done with relativistic BreitWigner functions that had a Flatté-type modification ${ }^{8}$ to accommodate the strong coupling to the $N \bar{K}$ state above that threshold, and assigned an isospin of either $I=$ 0 (solid and dashed black) or 1 (dotted black) so that the contributions from pure
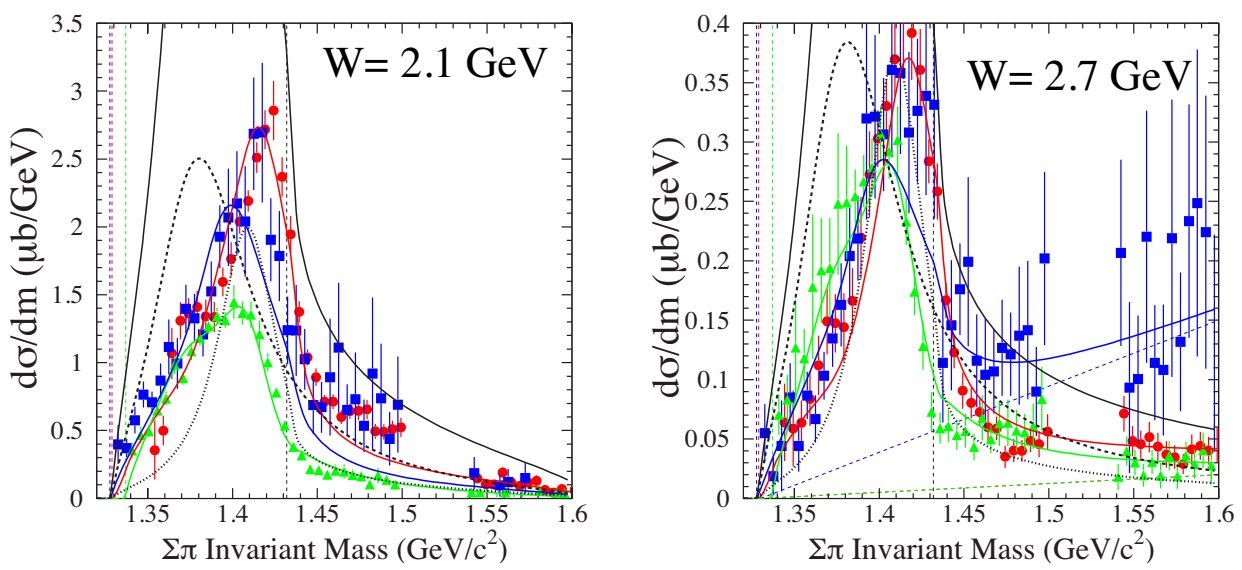

Fig. 1: Line shape of the $\Lambda(1405)$ for each $\Sigma \pi$ channel in two bins of $W$. The symbols are $\Sigma^{+} \pi^{-}$(red circles), $\Sigma^{0} \pi^{0}$ (blue squares), $\Sigma^{-} \pi^{+}$(green triangles). The data are from Ref. 5 , and the curves from Ref. 7 . The dashed vertical line shows the $N \bar{K}$ threshold, where a drop in the intensity is seen for all line shapes. 
isospin amplitudes could be accounted for in each $\Sigma \pi$ channel. The amplitudes were allowed to interfere coherently with each other, and reproduced the data well. ${ }^{9}$ While measurements of all three $\Sigma \pi$ line shapes represents great progress in the data from photoproduction, more data from other reactions are wanted since theory predicts that the population of the two poles of the $\Lambda(1405)$ depends on the reaction. ${ }^{2}$

\section{Cross Sections}

Measurements of the photoproduction differential cross sections of the $\Lambda(1405), \Sigma^{0}(1385)$, and $\Lambda(1520)$ were recently reported in Ref. 6 . We begin with the differential cross sections of the three $\Sigma \pi$ channels from the $\Lambda(1405)$. Due to the presence of higher mass states, the range of integration was fixed to be from $\Sigma \pi$ threshold up to a $\Sigma \pi$ mass of $1.5 \mathrm{GeV} / c^{2}$. Fig. 2 compares the three $\Sigma \pi$ channels for two $W$ bins, and are plotted against $\cos \theta_{K^{+}}^{\text {c.m. }}$. Fig. $2(\mathrm{a})$ is near production threshold, and we see stark qualitative differences between the three channels. This result was totally unanticipated, and further strengthens the case that there is a strong interference among different isospin channels near the $\Lambda(1405)$. If this is the case, this shows that the isospin interference is dependent not only on the c.m. energy, but also on the production angle of the $\Lambda(1405)$. It is interesting to note that this difference appears only in the near-production-threshold bins, where the difference in line shapes is prominent. Figure 2(b) is for a higher energy bin, and here we see that all three channels exhibit the same kind of forward-peaked behavior.

Next, we turn our attention to the comparison of the differential cross sections of the three hyperons. These results present first-time measurements of all three excited hyperons together within the same setup. Figures $3(\mathrm{a}-\mathrm{d})$ compare the differential cross sections of the three excited hyperons for different energies. The
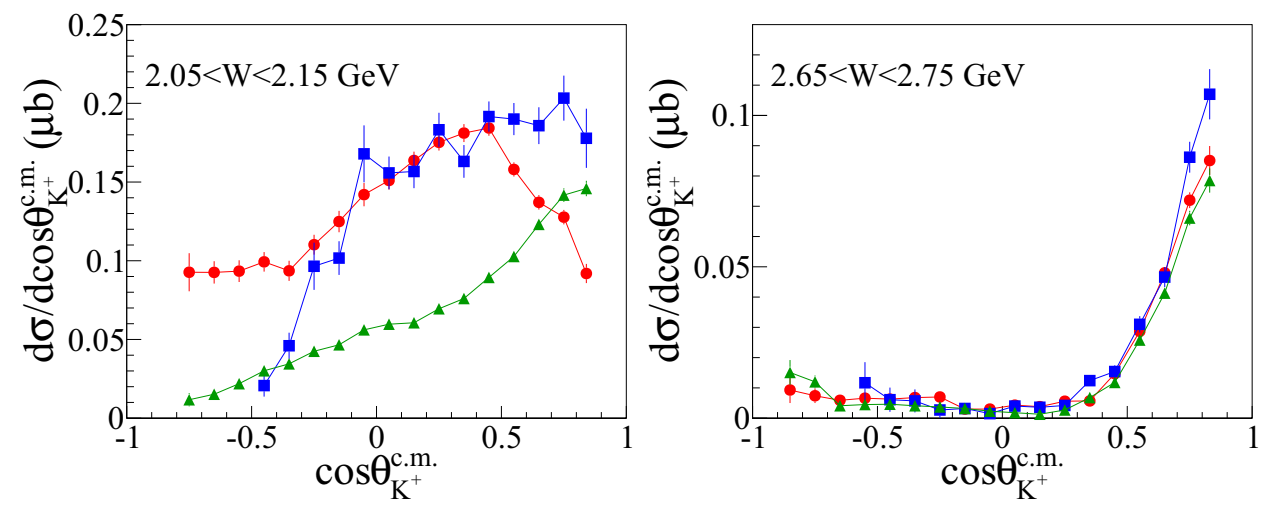

Fig. 2: Comparison of the differential cross sections for each $\Sigma \pi$ channel of the $\Lambda(1405)$. The data points for $\Sigma^{+} \pi^{-}$are shown with red circles, $\Sigma^{0} \pi^{0}$ with blue squares, and $\Sigma^{-} \pi^{+}$with green triangles. 

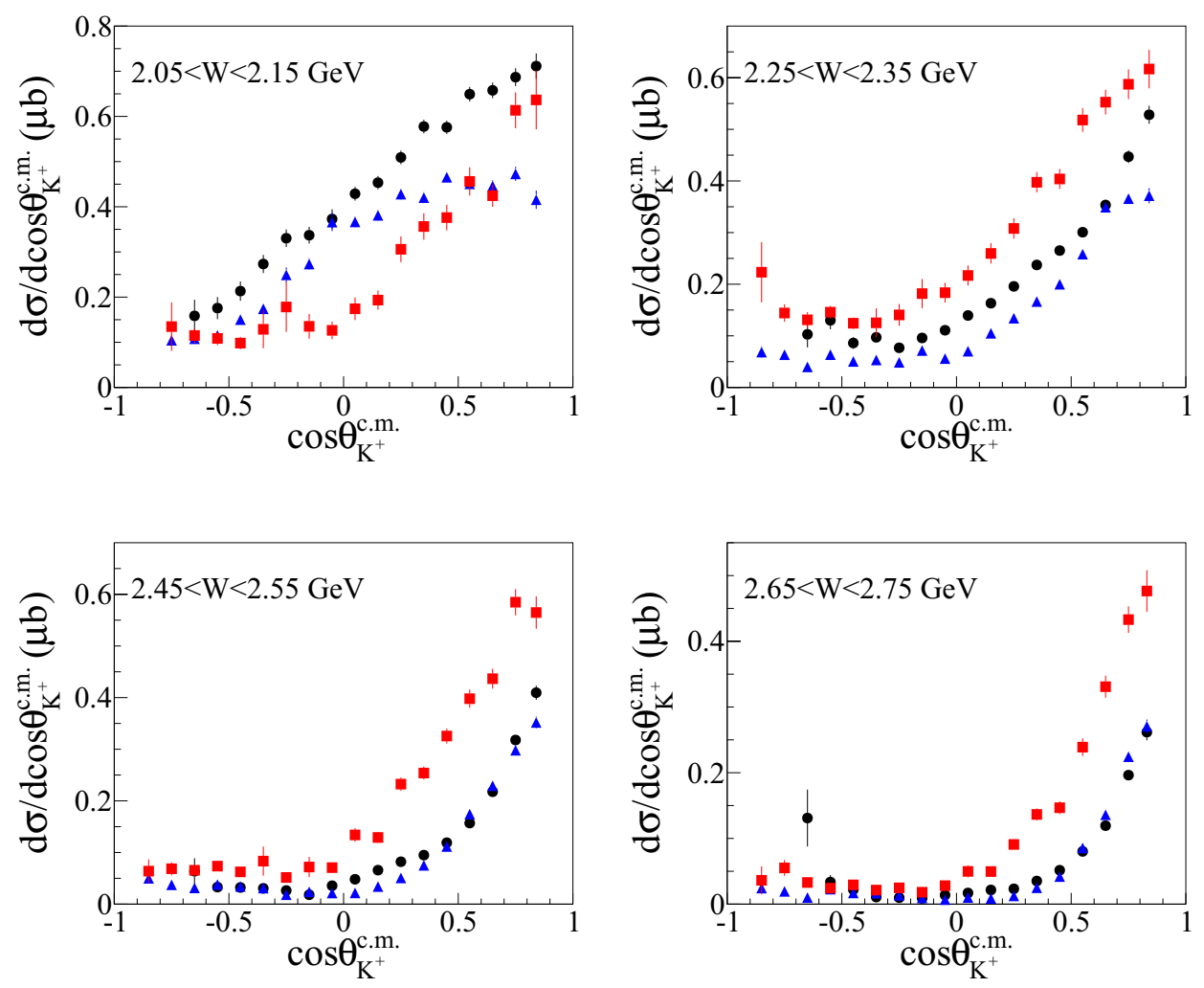

Fig. 3: Comparison of differential cross section for $\Lambda$ (1405) (blue triangles), $\Sigma^{0}(1385)$ (black circles), and $\Lambda(1520)$ (red squares) for four different $W$ bins.

$\Lambda(1405)$ is represented by the sum of all three measured $\Sigma \pi$ channels. The $\Sigma^{0}(1385)$ and $\Lambda(1520)$ have been corrected for the branching ratios to $\Lambda \pi(87.0 \%)$ and $\Sigma \pi$ (42\%), respectively. A comparison of the three differential cross sections shows that while the production of $\Lambda(1520)$ is suppressed near production threshold compared to the $\Lambda(1405)$ and $\Sigma^{0}(1385)$ due to its heavier mass, it has a larger cross section at higher energies. All three hyperons exhibit forward-peaked behavior at higher energies, suggesting that $K^{(*)}$-exchanges are the main production mechanisms for all states.

The differential cross sections were extrapolated to determine the total cross sections and are shown in Fig. 4, together with measurements for the ground state $K^{+} \Lambda$ and $K^{+} \Sigma^{0}$ from Ref. 10. We see that although the $Y^{*}$ cross sections are smaller than those for the ground state hyperons, there is still prominent production of the excited hyperons. Our binning in energy has been driven by having enough data within each bin to measure the $\Lambda(1405)$ line shape accurately, but further analyses of these cross sections may show couplings to intermediate $N^{*}$ states. 


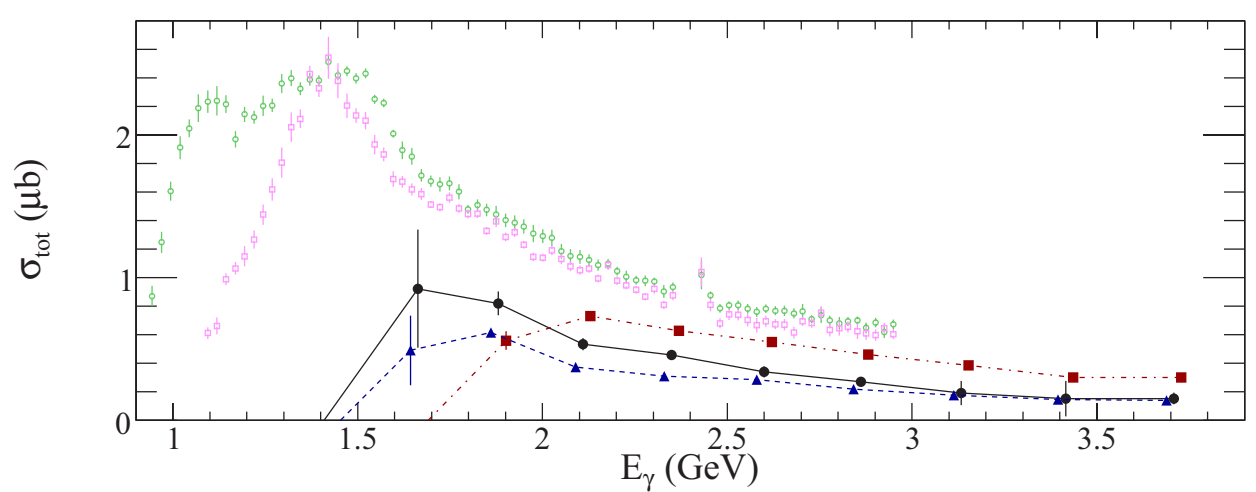

Fig. 4: Total cross section comparisons for $\Lambda$ (1405) (blue triangles), $\Sigma^{0}(1385)$ (black circles), and $\Lambda$ (1520) (red squares). For comparison, the total cross sections for $K^{+} \Lambda$ (open green circles) and $K^{+} \Sigma^{0}$ (open magenta squares) are shown.

\section{Future Plans with $12 \mathrm{GeV}$}

Further studies of strangeness will soon be possible at JLab, with many upgrade efforts ongoing. The accelerator is being upgraded to a maximum energy of $12 \mathrm{GeV}$, and a new experimental Hall D is being constructed, which will house the GlueX detector. The GlueX experiment ${ }^{11}$ will be a photoproduction experiment using a linearly polarized photon beam, with a hermetic detector for large angular coverage. The primary goal of GlueX will be to search for mesons with exotic quantum numbers which are not allowed in a simple $q \bar{q}$ model. This will be done by searching for and mapping out multiplets of mesons with specific quantum numbers, and comparing the resulting spectrum with that of theory. Recent results from lattice $\mathrm{QCD}^{12}$ show the existence of meson states that can be thought as having large constituent gluon components. Comparison with these results as well as other theories will validate whether our understanding of how QCD works at these energies is correct. Besides extensive searches for flavor multiplets of mesons, a rich physics program is possible due to the all-purpose detector, and this includes searches for excited $\Xi$ and $\Omega$ states. Installation of detectors in the hall has already started, and beam commissioning is scheduled to start towards the end of 2014. With the large statistics expected to be accumulated over years of running, there is potential for many new and exciting studies.

\section{Summary}

The photoproduced line shapes of the $\Lambda(1405)$ in all three $\Sigma \pi$ channels have been presented, as well as the differential cross sections of the $\Lambda(1405), \Sigma^{0}(1385)$, and $\Lambda(1520)$. The differential cross sections of the $\Lambda(1405)$ in each $\Sigma \pi$ channel have 
stark differences, which appear in near-production-threshold energies where the differences in line shapes are also prominent. The differential cross sections for all three excited hyperons exhibit a strong forward peak at higher energies, and the total cross sections are comparable to those of ground state hyperon production.

\section{Acknowledgments}

K. M. would like to thank the organizers of the NSTAR 2013 workshop for the invitation, hospitality, and financial support during the workshop. Financial support from the Jefferson Science Associates Travel Fund is gratefully acknowledged.

\section{References}

1. A. V. Anisovich, E. Klempt, V. A. Nikonov, A. V. Sarantsev and U. Thoma, "P-wave excited baryons from pion- and photo-induced hyperon production," Eur. Phys. J. A 47, 27 (2011), see also A. Sarantsev, these proceedings.

2. For a recent review, see T. Hyodo and D. Jido, "The nature of the $\Lambda(1405)$ resonance in chiral dynamics," Prog. Part. Nucl. Phys. 67, 55 (2012).

3. J. C. Nacher, E. Oset, H. Toki and A. Ramos, "Photoproduction of the $\Lambda(1405)$ on the proton and nuclei," Phys. Lett. B 455, 55 (1999).

4. I. Zychor et al., Phys. Lett. B 660, 167 (2008); M. Niiyama et al. [LEPS Collaboration], Phys. Rev. C 78035202 (2008); G. Agakishiev [HADES Collaboration], Phys. Rev. C85 035203 (2012), see also L. Fabbietti in these proceedings; Ref. 5; for a summary of recent theoretical fits to these results, see J. A. Oller, these proceedings.

5. K. Moriya et al. [CLAS Collaboration], "Measurement of the $\Sigma \pi$ photoproduction line shapes near the $\Lambda$ (1405)," Phys. Rev. C 87, 035206 (2013).

6. K. Moriya et al. [CLAS Collaboration], "Differential Photoproduction Cross Sections of the $\Sigma^{0}(1385), \Lambda(1405)$, and $\Lambda(1520)$," arXiv:1305.6776 [nucl-ex], submitted to Phys. Rev. C.

7. R. A. Schumacher and K. Moriya, "Isospin Decomposition of the Photoproduced $\Sigma \pi$ System Near the $\Lambda$ (1405)," arXiv:1303.0860 [nucl-ex], accepted by Nucl. Phys. A, http://dx.doi.org/10.1016/j.nuclphysa.2013.03.003.

8. S. M. Flatte, "Coupled-Channel Analysis of the $\pi \eta$ and $K \bar{K}$ Systems Near $K \bar{K}$ Threshold," Phys. Lett. B 63, 224 (1976). See Ref. 5 for implementation details.

9. An alternative fit for the $\Sigma^{0} \pi^{0}$ channel only using the full chiral unitary amplitudes was done in L. Roca and E. Oset, Phys. Rev. C 87, 055201 (2013), and also in arXiv:1307.5752 [nucl-th]. See also L. Roca, these proceedings.

10. R. Bradford et al. [CLAS Collaboration], "Differential cross sections for $\gamma+p \rightarrow$ $K^{+}+Y$ for $\Lambda$ and $\Sigma^{0}$ hyperons," Phys. Rev. C 73, 035202 (2006).

11. A. AlekSejevs et al. [GlueX Collaboration], "An initial study of mesons and baryons containing strange quarks with GlueX," arXiv:1305.1523[nucl-ex].

12. J. J. Dudek, "The lightest hybrid meson supermultiplet in QCD," Phys. Rev. D 84, 074023 (2011). 